\title{
Extended Hubbard Model in the Dimer Representation. I. Dimer Hamiltonian in the Large $U$ Limit
}

\author{
B. Grabiec and M. Matlak* \\ Institute of Physics, Silesian University \\ Uniwerstytecka 4, 40-007 Katowice, Poland
}

(Received December 14, 2001)

\begin{abstract}
We consider the extended Hubbard model for the single cubic lattice and rewrite it in the form of interacting dimers, using the exact solution of the dimer problem. We analytically derive the second quantization form of the dimer Hamiltonian eliminating from the considerations unoccupied dimer energy levels in the large $U$ limit (it is the only assumption). The resulting dimer Hamiltonian written with the use of the Hubbard operators and spin operators contains three terms, visualizing explicitly competing magnetic interactions (ferromagnetic, antiferromagnetic) as a generalization of the $t-J$ model. The presented, nonperturbative method, can in principle be applied to the cluster of any size (e.g. one central atom and $z$ its nearest neighbours). The use of the projection technique can further be applied in the case of a crystal to obtain the second quantization form of the extended Hubbard model for the sc lattice in the large $U$ limit.
\end{abstract}

PACS numbers: 71.10.-w, 71.10.Ca, 71.10.Fd

\section{Introduction}

The theoretical description of the electronic properties of solids always starts with an appropriate treatment of the electromagnetic interactions between charged particles (electrons + ions). Having in mind electronic subsystem we constantly deal with the Hubbard model itself, Ref. [1], or its extensions (many-band models, etc.), presented in a large number of monographs and review articles (see e.g. Refs. [2-13] and very large number of original papers cited therein). The impor-

*corresponding author; e-mail: matlak@server.phys.us.edu.pl 
tance of this approach and its place in the solid state theory lies in the fact that it is conceptually simplest and thus is a standard doorway to the correlated electron systems. It is commonly believed that the Hubbard model and its extensions can widely be used to explain many of the physical phenomena in quite different areas of the solid state physics: magnetic and transport properties of transition metals, their compounds and alloys, including insulator-to-metal transitions (cf. e.g. Refs. [1-13] and papers cited therein), high-temperature superconductors (negative $U$ models, cf. e.g. Ref. [14]), fluctuating valence systems and heavy fermions (Anderson-like models, Ref. [15], cf. also e.g. Refs. [11, 16]), liquid helium ${ }^{3} \mathrm{He}$ (cf. e.g. Refs. [17-19]), fullerenes (cf. e.g. Refs. [20-22]). It does not mean at all that the Hubbard model and its extensions are easy to handle and their relative mathematical simplicity is only an illusion. This fact is very well known in the literature. The situation is exceptionally difficult in the case of large $U$ (Coulomb repulsion). To demonstrate a high degree of complexity of the extended Hubbard model (a variety of different competing interactions within the model) we consider one-band electronic system described by the Hamiltonian

$$
\begin{aligned}
H= & \sum_{i \neq j, \sigma} t_{i, j} c_{i, \sigma}^{+} c_{j, \sigma}+U \sum_{i} n_{i, \uparrow} n_{i, \downarrow} \\
& +\frac{1}{2} \sum_{i \neq j, \sigma} J_{i j}^{(1)} n_{i, \sigma} n_{j, \sigma}+\frac{1}{2} \sum_{i \neq j, \sigma} J_{i j}^{(2)} n_{i, \sigma} n_{j,-\sigma} .
\end{aligned}
$$

The indices $(i, j)$ enumerate the lattice points, $t_{i j}$ is the hopping integral, $U$ denotes the intrasite Coulomb repulsion, $J^{(1)}$ and $J^{(2)}$ (generally, not necessary equal) describe the effective intersite Coulomb interactions, resulting from the original intersite Coulomb repulsion modified by polaronic effects (see e.g. Ref. [14] for details). The operators $c_{i, \sigma}\left(c_{i, \sigma}^{+}\right)$are the electronic annihilation (creation) operators in the lattice site $i$ with spin $\sigma(=\uparrow, \downarrow)$ and $n_{i, \sigma}=c_{i, \sigma}^{+} c_{i, \sigma}$. We restrict ourselves to the sc lattice and we apply tight binding approximation: $t_{[i, j]}=-t$, $J_{[i, j]}^{(1,2)}=J^{(1,2)}\left(U \gg \max \left\{\left|J^{(1)}\right|,\left|J^{(2)}\right|\right\}\right)$ where $i$ and $j$ are nearest neighbours. Using these assumptions we can rewrite the Hamiltonian (1) in an equivalent form dividing the original sc lattice into a set of interacting dimers (see Fig. 1).

We obtain

$$
\begin{aligned}
H= & \sum_{I} H_{I}^{\mathrm{D}}-t \sum_{I, \sigma}\left(c_{I, 2, \sigma}^{+} c_{I+1,1, \sigma}+c_{I+1,1, \sigma}^{+} c_{I, 2, \sigma}\right) \\
& -t \sum_{I \neq J, \sigma}\left(c_{I, 1, \sigma}^{+} c_{J, 1, \sigma}+c_{I, 2, \sigma}^{+} c_{J, 2, \sigma}\right)+J^{(1)} \sum_{I, \sigma} n_{I, 2, \sigma} n_{I+1,1, \sigma} \\
& +\frac{J^{(1)}}{2} \sum_{I \neq J, \sigma}\left(n_{I, 1, \sigma} n_{J, 1, \sigma}+n_{I, 2, \sigma} n_{J, 2, \sigma}\right)+J^{(2)} \sum_{I, \sigma} n_{I, 2, \sigma} n_{I+1,1,-\sigma} \\
& +\frac{J^{(2)}}{2} \sum_{I \neq J, \sigma}\left(n_{I, 1, \sigma} n_{J, 1,-\sigma}+n_{I, 2, \sigma} n_{J, 2,-\sigma}\right),
\end{aligned}
$$


where

$$
\begin{aligned}
H_{I}^{\mathrm{D}} & =-t \sum_{\sigma}\left(c_{I, 1, \sigma}^{+} c_{I, 2, \sigma}+c_{I, 2, \sigma}^{+} c_{I, 1, \sigma}\right)+U\left(n_{I, 1, \uparrow} n_{I, 1, \downarrow}+n_{I, 2, \uparrow} n_{I, 2, \downarrow}\right) \\
& +J^{(1)} \sum_{\sigma} n_{I, 1, \sigma} n_{I, 2, \sigma}+J^{(2)} \sum_{\sigma} n_{I, 1, \sigma} n_{I, 2,-\sigma} .
\end{aligned}
$$

The indices $(I, J)$ enumerate the dimers and $H_{I}^{\mathrm{D}}$ is the extended Hubbard model (1) of the $I$-th dimer. The second, fourth, and sixth term in (2) describe the hopping and effective Coulomb interactions between nearest dimers in the $z$-direction whereas the third, fifth, and seventh term represent the hopping and effective Coulomb interactions between nearest dimers $((y, z)$-plane) and between different dimer planes (cf. Fig. 1) ${ }^{\dagger}$ Starting from the exact solution of the dimer Hamiltonian (3) we can apply exactly the same procedure as for the Hubbard model itself, Ref. [23], leading to the final form of the dimer extended Hubbard Hamiltonian in the large $U$ limit (present paper). This approach is straightforward but very laborious. It differs significantly from the other approaches used in the literature as e.g. perturbation expansion or canonical transformation (cf. e.g. Refs. [24-32]) and ab initio derivations, Refs. [33-36], following the original approach from Refs. [37, 38] (see also Refs. $[39,11]$ and $[12]$ for a review). The application of the method presented in this paper to the crystal as a whole, described by the Hamiltonian (2) is given in the next paper (see Ref. [40]).

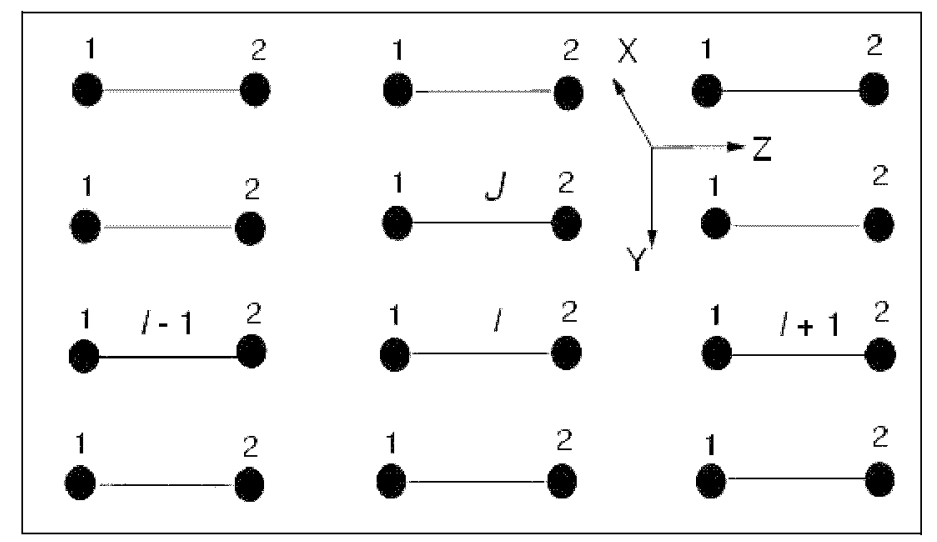

Fig. 1. A plane of the Hubbard dimers. The crystal is considered as a collection of parallel lying dimer planes.

\footnotetext{
${ }^{\dagger}$ The correctness of the decomposition of the original Hamiltonian (1) into a set of interacting dimers (2) can easily be verified using the usual Fourier transformation in the case of (1) and the dimer Fourier transformation $c_{I, 1(2), \sigma}=\frac{1}{\sqrt{N}} \sum_{k} c_{\boldsymbol{k}, \sigma} \mathrm{e}^{\mathrm{i} \boldsymbol{k} \cdot R_{I, 1(2)} c_{I, 1(2), \sigma}}$ in the case of (2) where $N$ is the number of lattice points. In both cases we obtain the same form of transformed Hamiltonian in the tight binding approximation.
} 


\section{Exact solution of the extended Hubbard model for a dimer}

The eigenvalue problem for the dimer Hamiltonian (3) can easily be found (we drop the dimer index $I$ ) using the standard procedure (cf. Refs. [41-43, 23]). Let us start with the vectors $\left|n_{1, \uparrow}, n_{1, \downarrow} ; n_{2, \uparrow}, n_{2, \downarrow}\right\rangle\left(n_{i, \sigma}=0,1 ; i=1,2 ; \sigma=\uparrow, \downarrow\right)$ forming the Fock basis of the dimer space of the states

$$
\begin{array}{rlrl}
|0\rangle=|0,0 ; 0,0\rangle, & |21\rangle=|1,1 ; 0,0\rangle, & & |31\rangle=|0,1 ; 1,1\rangle, \\
& |22\rangle=|1,0 ; 1,0\rangle, & & |32\rangle=|1,0 ; 1,1\rangle, \\
|11\rangle=|1,0 ; 0,0\rangle, & |23\rangle=|1,0 ; 0,1\rangle, & & |33\rangle=|1,1 ; 0,1\rangle, \\
|12\rangle=|0,1 ; 0,0\rangle, & |24\rangle=|0,1 ; 1,0\rangle, & |34\rangle=|1,1 ; 1,0\rangle, \\
|13\rangle=|0,0 ; 1,0\rangle, & |25\rangle=|0,1 ; 0,1\rangle, & & \\
|14\rangle=|0,0 ; 0,1\rangle, & |26\rangle=|0,0 ; 1,1\rangle, & |4\rangle=|1,1 ; 1,1\rangle .
\end{array}
$$

The first index in $|\alpha\rangle$ corresponds to $n=\sum_{i, \sigma} n_{i, \sigma}(=0,1,2,3,4)$, the second one (if necessary) enumerates the vectors corresponding to given $n$. It is very convenient to find first annihilation (creation) operators in the representation of the Fock space (4) when operating on this space. We find

$$
\begin{aligned}
c_{1, \uparrow} & =O_{0,11}+O_{12,21}+O_{13,22}+O_{14,23}+O_{26,32}+O_{25,33} \\
& +O_{24,34}+O_{31,4}, \\
c_{1, \downarrow} & =O_{0,12}-O_{11,21}+O_{13,24}+O_{14,25}+O_{26,31}-O_{23,33} \\
& -O_{22,34}-O_{32,4}, \\
c_{2, \uparrow} & =O_{0,13}-O_{11,22}-O_{12,24}+O_{14,26}-O_{25,31}-O_{23,32} \\
& +O_{21,34}+O_{33,4}, \\
c_{2, \downarrow} & =O_{0,14}-O_{11,23}-O_{12,25}-O_{13,26}+O_{24,31}+O_{22,32} \\
& +O_{21,33}-O_{34,4},
\end{aligned}
$$

where

$$
O_{\alpha, \beta}=|\alpha\rangle\langle\beta| .
$$

Inserting (5)-(8) into (3) we obtain the dimer Hamiltonian in the Fock space representation

$$
\begin{aligned}
H^{\mathrm{D}} & =-t\left(O_{11,13}-O_{21,24}+O_{23,26}-O_{33,31}+O_{13,11}-O_{24,21}+O_{26,23}-O_{31,33}\right. \\
& \left.+O_{12,14}+O_{21,23}-O_{24,26}-O_{34,32}+O_{14,12}-O_{23,21}-O_{26,24}-O_{32,34}\right) \\
& +U\left(O_{21,21}+O_{26,26}\right)+J^{(1)}\left(O_{22,22}+O_{25,25}\right)+J^{(2)}\left(O_{23,23}+O_{24,24}\right) \\
& +\left(U+J^{(1)}+J^{(2)}\right)\left(O_{31,31}+O_{32,32}+O_{33,33}+O_{34,34}\right) \\
& +2\left(U+J^{(1)}+J^{(2)}\right) O_{4,4}
\end{aligned}
$$


The operation with $H^{\mathrm{D}}$ given by (10) on the Fock basis (4) and using simple algebraic calculations we can easily find the eigenvalues $E_{\alpha}$ and eigenvectors $\left|E_{\alpha}\right\rangle$ of the dimer. This, in turn, allows to express the dimer Hamiltonian (3) (or (10)) in the form

$$
H^{\mathrm{D}}=\sum_{\alpha} E_{\alpha}\left|E_{\alpha}\right\rangle\left\langle E_{\alpha}\right|
$$

where

$$
\begin{array}{ll}
E_{0}=0, & \left|E_{0}\right\rangle=|0\rangle, \\
E_{11}=-t, & \left|E_{11}\right\rangle=\frac{1}{\sqrt{2}}(|11\rangle+|13\rangle), \\
E_{12}=t, & \left|E_{12}\right\rangle=\frac{1}{\sqrt{2}}(|11\rangle-|13\rangle), \\
E_{13}=-t, & \left|E_{13}\right\rangle=\frac{1}{\sqrt{2}}(|12\rangle+|14\rangle), \\
E_{14}=t, & \left|E_{14}\right\rangle=\frac{1}{\sqrt{2}}(|12\rangle-|14\rangle), \\
E_{21}=J^{(2)}, & \left|E_{21}\right\rangle=\frac{1}{\sqrt{2}}(|23\rangle+|24\rangle), \\
E_{22}=U, & \left|E_{22}\right\rangle=\frac{1}{\sqrt{2}}(|21\rangle-|26\rangle), \\
E_{23}=C+\frac{U+J^{(2)}}{2}, & \left|E_{23}\right\rangle=a_{1}(|21\rangle+|26\rangle)-a_{2}(|23\rangle-|24\rangle), \\
E_{24}=-C+\frac{U+J^{(2)}}{2}, & \left|E_{24}\right\rangle=a_{2}(|21\rangle+|26\rangle)+a_{1}(|23\rangle-|24\rangle), \\
E_{25}=J^{(1)}, & \left|E_{25}\right\rangle=|22\rangle, \\
E_{26}=J^{(1)}, & \left|E_{26}\right\rangle=|25\rangle, \\
E_{31}=t+U+J^{(1)}+J^{(2)}, & \left|E_{31}\right\rangle=\frac{1}{\sqrt{2}}(|31\rangle+|33\rangle), \\
E_{32}=-t+U+J^{(1)}+J^{(2)}, & \left|E_{32}\right\rangle=\frac{1}{\sqrt{2}}(|31\rangle-|33\rangle), \\
E_{33}=t+U+J^{(1)}+J^{(2)}, & \left|E_{33}\right\rangle=\frac{1}{\sqrt{2}}(|32\rangle+|34\rangle), \\
E_{34}=-t+U+J^{(1)}+J^{(2)}, & \left|E_{34}\right\rangle=\frac{1}{\sqrt{2}}(|32\rangle-|34\rangle), \\
E_{4}=2\left(U+J^{(1)}+J^{(2)}\right), & \left|E_{4}\right\rangle=|4\rangle
\end{array}
$$

and

$$
\begin{aligned}
& C=\sqrt{\left(\frac{U-J^{(2)}}{2}\right)^{2}+4 t^{2}}, \\
& a_{1}=\frac{1}{2} \sqrt{1+\frac{U-J^{(2)}}{2 C}}, \\
& a_{2}=\frac{1}{2} \sqrt{1-\frac{U-J^{(2)}}{2 C}} .
\end{aligned}
$$

The next important step in our calculations is the possibility to express the annihilation operators (5)-(8) as linear combinations of the transition operators between dimer states. This procedure can easily be performed when using the reciprocal relations to $(12)$ and inserting them into (5)-(8). We obtain 


$$
\begin{aligned}
& c_{1, \uparrow}=\frac{1}{\sqrt{2}}\left(P_{0,11}+P_{0,12}\right)+\frac{1}{\sqrt{2}} P_{11,25}-\frac{1}{\sqrt{2}} P_{12,25}+\frac{1}{2}\left(P_{13,21}+P_{13,22}\right) \\
& +\frac{1}{\sqrt{2}}\left(b P_{13,23}+a P_{13,24}\right)-\frac{1}{2}\left(P_{14,21}-P_{14,22}\right)+\frac{1}{\sqrt{2}}\left(a P_{14,23}-b P_{14,24}\right) \\
& +\frac{1}{2}\left(P_{21,33}-P_{21,34}\right)-\frac{1}{2}\left(P_{22,33}+P_{22,34}\right)+\frac{1}{\sqrt{2}}\left(a P_{23,33}+b P_{23,34}\right) \\
& -\frac{1}{\sqrt{2}}\left(b P_{24,33}-a P_{24,34}\right)+\frac{1}{\sqrt{2}}\left(P_{26,31}-P_{26,32}\right)+\frac{1}{\sqrt{2}} P_{31,4}+\frac{1}{\sqrt{2}} P_{32,4} \text {, } \\
& c_{1, \downarrow}=\frac{1}{\sqrt{2}}\left(P_{0,13}+P_{0,14}\right)+\frac{1}{\sqrt{2}} P_{13,26}-\frac{1}{\sqrt{2}} P_{14,26}+\frac{1}{2}\left(P_{11,21}-P_{11,22}\right) \\
& -\frac{1}{\sqrt{2}}\left(b P_{11,23}+a P_{11,24}\right)-\frac{1}{2}\left(P_{12,21}+P_{12,22}\right)-\frac{1}{\sqrt{2}}\left(a P_{12,23}-b P_{12,24}\right) \\
& -\frac{1}{2}\left(P_{21,31}-P_{21,32}\right)-\frac{1}{2}\left(P_{22,31}+P_{22,32}\right)+\frac{1}{\sqrt{2}}\left(a P_{23,31}+b P_{23,32}\right) \\
& -\frac{1}{\sqrt{2}}\left(b P_{24,31}-a P_{24,32}\right)-\frac{1}{\sqrt{2}}\left(P_{25,33}-P_{25,34}\right)-\frac{1}{\sqrt{2}} P_{33,4}-\frac{1}{\sqrt{2}} P_{34,4} \text {, } \\
& c_{2, \uparrow}=\frac{1}{\sqrt{2}}\left(P_{0,11}-P_{0,12}\right)-\frac{1}{\sqrt{2}} P_{11,25}-\frac{1}{\sqrt{2}} P_{12,25}-\frac{1}{2}\left(P_{13,21}+P_{13,22}\right) \\
& +\frac{1}{\sqrt{2}}\left(b P_{13,23}+a P_{13,24}\right)-\frac{1}{2}\left(P_{14,21}-P_{14,22}\right)-\frac{1}{\sqrt{2}}\left(a P_{14,23}-b P_{14,24}\right) \\
& -\frac{1}{2}\left(P_{21,33}+P_{21,34}\right)+\frac{1}{2}\left(P_{22,33}-P_{22,34}\right)+\frac{1}{\sqrt{2}}\left(a P_{23,33}-b P_{23,34}\right) \\
& -\frac{1}{\sqrt{2}}\left(b P_{24,33}+a P_{24,34}\right)-\frac{1}{\sqrt{2}}\left(P_{26,31}+P_{26,32}\right)+\frac{1}{\sqrt{2}} P_{31,4}-\frac{1}{\sqrt{2}} P_{32,4}, \\
& c_{2, \downarrow}=\frac{1}{\sqrt{2}}\left(P_{0,13}-P_{0,14}\right)-\frac{1}{\sqrt{2}} P_{13,26}-\frac{1}{\sqrt{2}} P_{14,26}-\frac{1}{2}\left(P_{11,21}-P_{11,22}\right) \\
& -\frac{1}{\sqrt{2}}\left(b P_{11,23}+a P_{11,24}\right)-\frac{1}{2}\left(P_{12,21}+P_{12,22}\right)+\frac{1}{\sqrt{2}}\left(a P_{12,23}-b P_{12,24}\right) \\
& +\frac{1}{2}\left(P_{21,31}+P_{21,32}\right)+\frac{1}{2}\left(P_{22,31}-P_{22,32}\right)+\frac{1}{\sqrt{2}}\left(a P_{23,31}-b P_{23,32}\right) \\
& -\frac{1}{\sqrt{2}}\left(b P_{24,31}+a P_{24,32}\right)+\frac{1}{\sqrt{2}}\left(P_{25,33}+P_{25,34}\right)-\frac{1}{\sqrt{2}} P_{33,4}+\frac{1}{\sqrt{2}} P_{34,4},
\end{aligned}
$$

where

$$
P_{\alpha, \beta}=\left|E_{\alpha}\right\rangle\left\langle E_{\beta}\right|
$$

and

$$
a=a_{1}+a_{2}, \quad b=a_{1}-a_{2} .
$$

In this way we have expressed the annihilations operators $c_{1(2), \sigma}$ in the dimer representation. This, in turn, allows to derive later the extended Hubbard model for a dimer in the large $U$ limit using the projection technique. 


\section{Large $U$ limit for a dimer}

Looking at the eigenvalues of the extended Hubbard model for a dimer (12) we can see that in the large $U$ limit $\left(U \gg t, U \gg \max \left\{\left|J^{(1)}\right|,\left|J^{(2)}\right|\right\}\right.$ the energies $E_{\alpha}=E_{22}, E_{23}, E_{31}, E_{32}, E_{33}, E_{34}$ and $E_{4}$ take on large, positive values, much larger than the other, producing in the partition function the terms which can practically be neglected. It means that the mentioned energies cannot be occupied by electrons in a reasonable temperature range $(1 \mathrm{eV} \approx 11604.5 \mathrm{~K})$ and therefore can be excluded from our considerations. The dimer Hamiltonian (11) in the large $U$ limit can thus be reduced to the following expression:

$$
\begin{aligned}
\bar{H}^{\mathrm{D}} & =-t\left(P_{11,11}-P_{12,12}+P_{13,13}-P_{14,14}\right)+J^{(2)} P_{21,21} \\
& +\left(-C+\frac{U+J^{(2)}}{2}\right) P_{24,24}+J^{(1)}\left(P_{25,25}+P_{26,26}\right) .
\end{aligned}
$$

The Hamiltonian (22) can easily be written in the compact second quantization form. Let us first introduce the Hubbard operators

$$
a_{i, \sigma}=c_{i, \sigma}\left(1-n_{i,-\sigma}\right), \quad b_{i, \sigma}=c_{i, \sigma} n_{i,-\sigma}
$$

and spin operators

$$
\begin{aligned}
& S_{i}^{z}=\frac{1}{2}\left(n_{i, \uparrow}-n_{i, \downarrow}\right)=\frac{1}{2}\left(n_{i, \uparrow}^{a}-n_{i, \downarrow}^{a}\right), \\
& S_{i}^{+}=c_{i, \uparrow}^{+} c_{i, \downarrow}=a_{i, \uparrow}^{+} a_{i, \downarrow}, \quad S_{i}^{-}=c_{i, \downarrow}^{+} c_{i, \uparrow}=a_{i, \downarrow}^{+} a_{i, \uparrow},
\end{aligned}
$$

where $n_{i, \sigma}^{a}=a_{i, \sigma}^{+} a_{i, \sigma}(i=1,2, \sigma=\uparrow, \downarrow)$. To demonstrate how to rewrite (22) in the second quantization form let us consider the first term in (22). Applying (20) and (12) we can rewrite this term in the equivalent form

$$
\begin{aligned}
& -t\left(P_{11,11}-P_{12,12}+P_{13,13}-P_{14,14}\right) \\
& \quad=-t\left(O_{11,13}+O_{13,11}+O_{12,14}+O_{14,12}\right) .
\end{aligned}
$$

According to (9) and (4) we get

$$
\begin{aligned}
& O_{11,13}=|1,0 ; 0,0\rangle\langle 0,0 ; 1,0| \equiv c_{1, \uparrow}^{+}\left(1-n_{1, \downarrow}\right) c_{2, \uparrow}\left(1-n_{2, \downarrow}\right)=a_{1, \uparrow}^{+} a_{2, \uparrow}, \\
& O_{13,11}=a_{2, \uparrow}^{+} a_{1, \uparrow}, \\
& O_{12,14}=|0,1 ; 0,0\rangle\langle 0,0 ; 0,1| \equiv c_{1, \downarrow}^{+}\left(1-n_{1, \uparrow}\right) c_{2, \downarrow}\left(1-n_{2, \uparrow}\right)=a_{1, \downarrow}^{+} a_{2, \downarrow}, \\
& O_{14,12}=a_{2, \downarrow}^{+} a_{1, \downarrow} .
\end{aligned}
$$

Thus, the first term in (22) can be written in the second quantization form to be

$$
-t\left(P_{11,11}-P_{12,12}+P_{13,13}-P_{14,14}\right)=-t \sum_{\sigma}\left(a_{1, \sigma}^{+} a_{2, \sigma}+a_{2, \sigma}^{+} a_{1, \sigma}\right) .
$$


Exactly the same procedure can be applied to other terms in (22). It gives the result (we introduce here (23) and (24)):

$$
\begin{aligned}
\bar{H}^{\mathrm{D}}= & -t \sum_{\sigma}\left[a_{1, \sigma}^{+} a_{2, \sigma}+a_{2, \sigma}^{+} a_{1, \sigma}\right]+2 J^{(1)}\left(S_{1}^{z} \cdot S_{2}^{z}+\frac{n_{1}^{a} n_{2}^{a}}{4}\right) \\
& -2 J^{(2)}\left(S_{1}^{z} \cdot S_{2}^{z}-\frac{n_{1}^{a} n_{2}^{a}}{4}\right)+\left[\frac{4 x t}{\left(1-x^{(2)}\right)^{2} R}+J^{(2)}\left(1-\frac{(1+R)^{2}}{4 R}\right)\right] \\
& \times\left[\boldsymbol{S}_{1} \cdot S_{2}-\frac{n_{1}^{a} n_{2}^{a}}{4}\right]+\frac{x t}{\left(1-x^{(2)}\right)^{2} R}\left[\frac{1-R}{1+R}+x^{(2)}\right] \\
& \times\left[2\left(b_{1, \uparrow}^{+} a_{1, \downarrow}^{+} a_{2, \downarrow} b_{2, \uparrow}+b_{2, \uparrow}^{+} a_{2, \downarrow}^{+} a_{1, \downarrow} b_{1, \uparrow}\right)+\left(1-n_{1}^{a}\right) n_{2}^{b}+\left(1-n_{2}^{a}\right) n_{1}^{b}-n_{1}^{b} n_{2}^{b}\right] \\
& +\frac{t}{2\left(1-x^{(2)}\right)}\left[\frac{1+x^{(2)}}{R}+x^{(2)}-1\right] \sum_{\alpha=1,2} \sum_{\sigma}\left(a_{\alpha, \sigma}^{+} b_{\bar{\alpha}, \sigma}+b_{\alpha, \sigma}^{+} a_{\bar{\alpha}, \sigma}\right), \quad(28)
\end{aligned}
$$

where

$$
\begin{aligned}
& x=\frac{t}{U}, \quad x^{(2)}=\frac{J^{(2)}}{U}, \\
& R=\sqrt{1+\frac{16 x^{2}}{\left(1-x^{(2)}\right)^{2}}}, \\
& n_{1(2)}^{a(b)}=\sum_{\sigma} n_{1(2), \sigma}^{a(b)} \quad\left(n_{1(2)}^{b}=b_{1(2), \sigma}^{+} b_{1(2), \sigma}=n_{1(2), \sigma} n_{1(2),-\sigma}\right)
\end{aligned}
$$

and $\bar{\alpha}=1$ (2) if $\alpha=2$ (1). The Hamiltonian (28) is exactly equivalent to (22). We see that the first three terms in (28) represent the exact form of the dimer Hamiltonian (3) in the limit $U \rightarrow \infty$. Besides, the dimer Hamiltonian (28) contains three competing magnetic (ferromagnetic and antiferromagnetic) interactions of the Ising- and Heisenberg-type. The complicated form of the Hamiltonian (28) can essentially be reduced when applying the linear Taylor expansion of the coefficients with respect to $x$ and $x^{(2)}\left(x, x^{(2)} \ll 1\right)$. We obtain

$$
\begin{aligned}
\bar{H}^{\mathrm{D}}= & -t \sum_{\sigma}\left[a_{1, \sigma}^{+} a_{2, \sigma}+a_{2, \sigma}^{+} a_{1, \sigma}\right]+2 J^{(1)}\left(S_{1}^{z} S_{2}^{z}+\frac{n_{1}^{a} n_{2}^{a}}{4}\right) \\
& -2 J^{(2)}\left(S_{1}^{z} S_{2}^{z}-\frac{n_{1}^{a} n_{2}^{a}}{4}\right)+\frac{4 t^{2}}{U}\left[\boldsymbol{S}_{1} \cdot \boldsymbol{S}_{2}-\frac{n_{1}^{a} n_{2}^{a}}{4}\right] .
\end{aligned}
$$

We can see that for $J^{(1)}=J^{(2)}=0$ the Hamiltonian (31) reduces to the well-known $t-J$ model (cf. Ref. [37]) in the case of the Hubbard dimer. 


\section{Projection technique}

Exactly the same result (28) can be received by applying a more general approach. Let us first note that after the elimination of the unoccupied dimer energy levels the subspace of the dimer eigenvectors (12) in the large $U$ limit contains the following eigenvectors: $\left|E_{0}\right\rangle,\left|E_{11}\right\rangle,\left|E_{12}\right\rangle,\left|E_{13}\right\rangle,\left|E_{14}\right\rangle,\left|E_{21}\right\rangle,\left|E_{24}\right\rangle,\left|E_{25}\right\rangle$ and $\left|E_{26}\right\rangle$. Thus, the projection operator onto this subspace has the form

$$
\begin{aligned}
P= & P_{0,0}+P_{11,11}+P_{12,12}+P_{13,13}+P_{14,14} \\
& +P_{21,21}+P_{24,24}+P_{25,25}+P_{26,26} .
\end{aligned}
$$

The second quantization form of this operator can be obtained in exactly the same way as the formula (27). Each of the transition operators $P_{\alpha, \beta}$ in (32) can be written as a linear combination of the operators $O_{\alpha, \beta}$ (see (9)) by using (12) which, in turn, can directly be rewritten in the second quantization, similar to $(27)$. In this way we get

$$
\begin{aligned}
& P=1-\frac{1}{2}\left(n_{1}^{b}+n_{2}^{b}\right)+\frac{1}{4} n_{1}^{b} n_{2}^{b}+\frac{1}{8}\left(1-\frac{1}{R}\right)\left[4\left(\boldsymbol{S}_{1} \cdot \boldsymbol{S}_{2}-\frac{1}{4} n_{1}^{a} n_{2}^{a}\right)\right. \\
& \left.+2\left(b_{1, \uparrow}^{+} a_{1, \downarrow}^{+} a_{2, \downarrow} b_{2, \uparrow}+b_{2, \uparrow}^{+} a_{2, \downarrow}^{+} a_{1, \downarrow} b_{1, \uparrow}\right)+\left(1-n_{1}^{a}\right) n_{2}^{b}+\left(1-n_{2}^{a}\right) n_{1}^{b}-n_{1}^{b} n_{2}^{b}\right] \\
& \quad+\frac{x}{\left(1-x^{(2)}\right) R} \sum_{\alpha=1,2} \sum_{\sigma}\left(a_{\alpha, \sigma}^{+} b_{\bar{\alpha}, \sigma}+b_{\alpha, \sigma}^{+} a_{\bar{\alpha}, \sigma}\right),
\end{aligned}
$$

where $x, x^{(2)}$, and $R$ are given by (29) and (30), respectively. With the use of the projection operator $P$ the dimer Hamiltonian $\bar{H}^{\mathrm{D}}$ can actually be written in the form

$$
\bar{H}^{\mathrm{D}}=P H^{\mathrm{D}} P .
$$

Applying the explicit form of the dimer Hamiltonian (3) and the formula (34) we can write

$$
\begin{aligned}
\bar{H}^{\mathrm{D}}= & -t \sum_{\sigma}\left(\bar{c}_{2, \sigma}^{+} \bar{c}_{1, \sigma}+\bar{c}_{1, \sigma}^{+} \bar{c}_{2, \sigma}\right)+U\left(\bar{n}_{1, \uparrow}^{+} \bar{n}_{1, \downarrow}+\bar{n}_{2, \uparrow}^{+} \bar{n}_{2, \downarrow}\right) \\
& +J^{(1)} \sum_{\sigma} \bar{n}_{1, \sigma}^{+} \bar{n}_{2, \sigma}+J^{(2)} \sum_{\sigma} \bar{n}_{1, \sigma}^{+} \bar{n}_{2,-\sigma},
\end{aligned}
$$

where $(i=1,2 ; \sigma=\uparrow, \downarrow)$

$$
\begin{aligned}
& \bar{c}_{i, \sigma}=c_{i, \sigma} P \equiv P c_{i, \sigma} P, \quad \bar{c}_{i, \sigma}^{+}=P c_{i, \sigma}^{+}=\left(c_{i, \sigma} P\right)^{+} \equiv P c_{i, \sigma}^{+} P, \\
& \bar{n}_{i, \sigma}=n_{i, \sigma} P, \quad \bar{n}_{i, \sigma}^{+}=P n_{i, \sigma} .
\end{aligned}
$$

The most simple form of these operators with the use of (23), (24) and (33) reads

$$
\bar{c}_{1, \uparrow}=\underline{\underline{a}}_{1, \uparrow}+\beta\left[S_{2}^{z} a_{1, \uparrow}+S_{2}^{-} a_{1, \downarrow}\right]-\delta\left[S_{1}^{z} a_{2, \uparrow}+S_{1}^{-} a_{2, \downarrow}\right],
$$




$$
\bar{c}_{1, \downarrow}=\underline{\underline{a}}_{1, \downarrow}-\beta\left[S_{2}^{z} a_{1, \downarrow}-S_{2}^{+} a_{1, \uparrow}\right]+\delta\left[S_{1}^{z} a_{2, \downarrow}-S_{1}^{+} a_{2, \uparrow}\right]
$$

and

$$
\begin{aligned}
& \bar{n}_{1, \uparrow}=\left(1-\beta \frac{n_{2}^{a}}{2}-\frac{n_{2}^{b}}{2}\right) S_{1}^{z}+\beta \frac{n_{1}^{a}}{2} S_{2}^{z}+\beta\left(S_{1}^{z} S_{2}^{z}+S_{1}^{+} S_{2}^{-}\right)+\frac{n_{1}^{a}}{2}\left(1-\frac{n_{2}^{b}}{2}\right) \\
&+ \beta\left[\frac{1}{2} n_{1}^{b}\left(1-n_{2}^{a}-\frac{n_{2}^{b}}{2}\right)-\frac{n_{1}^{a} n_{2}^{a}}{4}+b_{1, \uparrow}^{+} a_{1, \downarrow}^{+} a_{2, \downarrow} b_{2, \uparrow}\right] \\
&+ \delta\left(a_{1, \uparrow}^{+} b_{2, \uparrow}+a_{2, \downarrow}^{+} b_{1, \downarrow}+b_{1, \uparrow}^{+} a_{2, \uparrow}+b_{1, \downarrow}^{+} a_{2, \downarrow}\right), \\
& \bar{n}_{1, \downarrow}=-\left(1-\beta \frac{n_{2}^{a}}{2}-\frac{n_{2}^{b}}{2}\right) S_{1}^{z}-\beta \frac{n_{1}^{a}}{2} S_{2}^{z}+\beta\left(S_{1}^{z} S_{2}^{z}+S_{1}^{-} S_{2}^{+}\right)+\frac{n_{1}^{a}}{2}\left(1-\frac{n_{2}^{b}}{2}\right) \\
&+\beta {\left[\frac{1}{2} n_{1}^{b}\left(1-n_{2}^{a}-\frac{n_{2}^{b}}{2}\right)-\frac{n_{1}^{a} n_{2}^{a}}{4}+b_{1, \uparrow}^{+} a_{1, \downarrow}^{+} a_{2, \downarrow} b_{2, \uparrow}\right] } \\
&+\delta\left(a_{1, \downarrow}^{+} b_{2, \downarrow}+a_{2, \uparrow}^{+} b_{1, \uparrow}+b_{1, \uparrow}^{+} a_{2, \uparrow}+b_{1, \downarrow}^{+} a_{2, \downarrow}\right), \\
& \underline{a}_{1(2), \sigma}=\underline{a}_{1(2), \sigma}+\beta\left(\underline{b}_{1(2), \sigma}+a_{1(2),-\sigma}^{+} a_{2(1),-\sigma} b_{2(1), \sigma}\right) \\
&+\delta\left[\underline{b}_{2(1), \sigma}+a_{2(1),-\sigma}^{+} a_{1(2),-\sigma} b_{1(2), \sigma}+a_{2(1), \sigma} \frac{n_{1(2)}^{a}}{2}\right] \\
& \beta \\
& \underline{a}_{1(2), \sigma}=a_{1(2), \sigma}\left(1-\frac{\beta}{2} n_{2(1)}^{a}-\frac{1}{2} n_{2(1)}^{b}\right), \\
& \underline{b}_{1(2), \sigma}=b_{1(2), \sigma}\left(1-n_{2(1)}^{a}-\frac{1}{2} n_{2(1)}^{b}\right), \\
& \frac{1}{4}\left(1-\frac{1}{R}\right), \quad \delta=\frac{x}{\left(1-x{ }^{(2)}\right) R},
\end{aligned}
$$

where $x, x^{(2)}$, and $R$ are defined by (29) and (30). The expressions for $\bar{c}_{2, \sigma}$ and $\bar{n}_{2, \sigma}(\sigma=\uparrow, \downarrow)$ can easily be found by changing $1 \leftrightarrow 2$ in (37), (38) and (39), (40). It is clear that inserting (37)-(40) into (35) we obtain exactly the same expression for the dimer Hamiltonian in the large $U$ limit (28) when performing straightforward but very laborious algebraic calculations.

\section{Conclusion}

Starting from the exact spectrum of the extended Hubbard model for a dimer (the smallest complex of interacting atoms) and rejecting from the considerations unoccupied energy levels we were able to derive the second quantization form of the dimer Hamiltonian in the large $U$ limit (Sec. 3). This derivation was straightforward without applying perturbation expansion or canonical transformation. In principle, the presented method can be applicable to any size of a finite complex of interacting atoms as e.g. to a cluster with one central atom and $z$ its nearest 
neighbours and to any other models with one model parameter (or more) substantially greater than the other. The only complication which here arises is the fact that the mathematical complexity of the problem grows exponentially up with the size of the cluster (see e.g. Refs. [44-47]). In Sec. 4 we have shown that exactly the same result (28) can be obtained with the use of the projection technique. This method applied to the dimer is nothing else as "redefining" the primary construction operators in (3) and replacing all of them by (37)-(40) in the large $U$ limit. In the next paper [40] we will show that the method is also applicable to the case of a crystal when one divides the Hamiltonian of the original lattice into a set of interacting dimers (2) and uses a modified projection procedure, introduced in Sec. 4.

\section{References}

[1] J. Hubbard, Proc. R. Soc. Lond. A 276, 238 (1963); ibid 281, 401 (1964).

[2] C. Herring, in: Magnetism, Vol. IV, Eds. G.T. Rado and H. Suhl, Adademic Press, New York 1966, p. 1.

[3] J. Friedel, in: The Physics of Metals, Ed. J.M. Ziman, Cambridge University Press, Cambridge 1969, p. 340.

[4] B. Brandow, Adv. Phys. 26, 651 (1977).

[5] T. Moriya, Spin Fluctuations in Itinerant Electron Magnetism, Springer Series in Solid States Sciences, Vol. 56, Springer, Berlin 1985.

[6] W. Nolting, Quantentheorie des Magnetismus, Vol. 1 and 2, Teubner, Stuttgart 1986.

[7] Metalic Magnetism, Ed. H. Capelmann, Springer Topics in Current Physics, Vol. 42, Springer, Berlin 1987.

[8] N.F. Mott, Metal-Insulator Transitions, Taylor and Francis, London 1990.

[9] P.A. Cox, Transitions Metal Oxides, International Series of Monographs on Chemistry, Vol. 27, Clarendon Press, Oxford 1992.

[10] D.E. Logan, Y.H. Szczech, M.E. Tusch, in: Metal-Insulator Transitions Revisited, Eds. P.P. Edwards, C.N.R. Rao, Taylor and Francis, London 1995, p. 395.

[11] P. Fulde, Electron Correlations in Molecules and Solids, Springer, Berlin 1995.

[12] F. Gebhard, The Mott Metal-Insulator Transitions, Springer Tracts in Modern Physics, Vol. 137, Springer, Berlin 1997.

[13] M. Imada, A. Fujimori, Rev. Mod. Phys. 70, 1039 (1998).

[14] R. Micnas, J. Ranninger, S. Robaszkiewicz, Rev. Mod. Phys. 62, 113 (1990).

[15] P.W. Anderson, Phys. Rev. 124, 41 (1961).

[16] C. Varma, Y. Yafet, Phys. Rev. B 13, 2950 (1976).

[17] D. Vollhardt, Rev. Mod. Phys. 56, 99 (1984).

[18] D. Vollhardt, P.Wölfle, P.W. Anderson, Phys. Rev. B 35, 6703 (1987).

[19] D. Vollhardt, P. Wöltle, The Superfluid Phases of Helium-3, Taylor and Francis, London 1990. 
[20] Electronic Properties of Fullerenes, Eds. H. Kuzmany, J. Fink, M. Mehring, S. Roth, Springer Series in Solid State Sciences, Vol. 117, Springer, Berlin 1993.

[21] S. Chakravarty, M.P. Gelfand, S. Kivelson, Science 254, 970 (1991).

[22] S.L. Sondhi, M.P. Gelfand, H.Q. Lin, D.K. Campbell, Phys. Rev. B 51, 5943 (1995).

[23] M. Matlak, J. Aksamit, B. Grabiec, W. Nolting, submitted to J. Phys. C.

[24] D.J. Klein, W.A. Seitz, Phys. Rev. B 8, 2236 (1973).

[25] J. Florencio, Jr, K.A. Chao, Phys. Rev. Lett. 35, 741 (1975).

[26] W. Kohn, Phys. Rev. 133, 171 (1964).

[27] A.B. Harris, R.V. Lange, Phys. Rev. 157, 295 (1967).

[28] T.A. Kaplan, R.A. Bari, J. Appl. Phys. 41, 875 (1970).

[29] J. Florencio, Jr, K.A. Chao, Phys. Rev. B 14, 3121 (1976).

[30] K.A. Chao, J. Spałek, A.M. Oleś, J. Phys. C 10, L271 (1977); Phys. Status Solidi B 84, 747 (1977); Phys. Rev. B 18, 3453 (1980).

[31] J. Spałek, A.M. Oleś, Physica B 86-88, 375 (1977).

[32] J. Spałek, K.A. Chao, J. Phys. C 13, 5241 (1980).

[33] V.Yu. Irkhin, M.I. Katsnelson, J. Phys. C 18, 4173 (1985).

[34] V.Yu. Irkhin, Yu.P. Irkhin, Phys. Status Solidi B 183, 9 (1994).

[35] H. Eskes, R. Eder, Phys. Rev. B 54, R14226 (1996).

[36] V.Yu. Irkhin, Phys. Rev. B 57, 13375 (1998).

[37] P.W. Anderson, Phys. Rev. 115, 2 (1959).

[38] P.W. Anderson, in: Solid State Physics, Eds. F. Seitz, D. Turnbull, Vol. 14, Academic Press, New York 1963, p. 99.

[39] E. Dagotto, Rev. Mod. Phys. 66, 763 (1994).

[40] B. Grabiec, M. Matlak, Acta Phys. Pol. A 101, (2002).

[41] V.C. Cheng, S.H. Chen, Physica B 85, 299 (1977).

[42] M. Matlak, Phys. Status Solidi B 99, K87 (1980).

[43] B. Lorentz, Phys. Status Solidi B 119, 555 (1983).

[44] L.M. Falicov, R.H. Victor, Phys. Rev. B 30, 1695 (1984).

[45] J. Callaway, D.P. Chen, R. Tang, Phys. Rev. B 35, 3705 (1987).

[46] J. Callaway, D.P. Chen, Y. Zhang, Phys. Rev. B 36, 2084 (1987).

[47] G.M. Pastor, R. Hirsch, B. Mühlschlegel, Phys. Rev. B 53, 10382 (1996). 